\title{
GETTYSBURG, UN LIEU DE MEMOIRE AMERICAIN
}

Alain Reyniers ${ }^{1}$

En reprenant la lecture de la sixième leçon donnée par Jan Baetens au Département de communication, je confesse avoir vécu l'angoisse de la page blanche. Que dire sur un texte dont un parcours précédent m'avait déjà amené à en partager les idées, à en savourer l'érudition, à en reconnaitre le bien fondé du propos ? Comment aller contre l'idée qu'il n'y a pas de mémoire sans oubli ni sélection, pas de vie commune sans passé partagé, pas de passé sans construction - tout au moins en partie - de celui-ci ? L'approche actuelle de la politique patrimoniale souffrirait d'un relâchement par rapport à l'exigence chronologique et à la contextualisation précise des faits du passé. Elle serait l'alibi d'enjeux économiques et idéologiques, privilégiant la mémoire du lieu plutôt que celle des personnes. Le recours massif à la digitalisation et aux nouveaux médias ne rendrait pas mieux le passé pour autant et l'expérience de ce dernier se traduirait moins en acquisition de connaissances qu'en opportunité supplémentaire de consommation esthétique.

C'est en méditant ces idées que me sont peu à peu venues à l'esprit des bruits, des saveurs, des images des souvenirs d'abord vagues et imprécis, par bribes, entremêlés, puis prenant peu à peu plus de consistance. Des gens, des milliers de visiteurs, de tous âges, pressés ou

1 Alain Reyniers est professeur au Département de communication de l'UCL.

Recherches en communication, $\mathrm{n}^{\circ} 31$ (2009). 
décontractés, des familles, des groupes scolaires ou du troisième âge, assis sur quelques bancs, déambulant en file indienne ou en essaims compacts, parcourant pendant des heures un itinéraire muséal balisé de vitrines et de salles de projection audio-visuelle. Des promeneurs également, parcourant des kilomètres de voierie reliant des zones urbaines, rurales et forestières parsemées de vieux canons et de monuments. C'était durant l'été 2009, à Gettysburg, dans l'Etat de Pennsylvanie, aux USA.

\section{La bataille de Gettysburg}

Le site de Gettysburg n'est probablement pas très connu en dehors des Etats-Unis. C'est pourtant l'un des principaux lieux de mémoire de l'histoire nord américaine. Là-bas se déroula l'un des évènements majeurs de la Guerre de Sécession ${ }^{1}$. Durant trois jours, entre le $1^{\text {er }}$ et le 3 juillet 1863, l'armée sudiste des Confédérés de Virginie, commandée par le général Robert E. Lee, affronta les soldats de l'Union dirigés par le général George Gordon Meade. La bataille, longtemps indécise, se solda par la victoire des troupes du Nord sur celles du Sud, au prix d'une boucherie effroyable: plus de 51.000 soldats des deux camps (28.000 Sudistes et 23.000 Nordistes) y laissèrent leur vie, y furent blessés ou y disparurent.

L'issue de cette bataille constitua un tournant dans le conflit qui opposait le Nord au Sud depuis le mois de janvier 1861. Jusque-là, les Confédérés avaient plutôt conservé l'initiative et accumulaient les victoires. Avec cette victoire-ci, le Nord entrevoyait enfin une issue du conflit qui puisse être à son avantage. Il lui faudra pourtant encore deux années de durs combats avant d'en finir avec la guerre civile.

1 La Guerre de Sécession, mieux connue aux Etats-Unis comme « American Civil War », se déroula entre 1861 et 1865 . Elle est souvent présentée comme une guerre entre des Etats du Sud esclavagistes et des Etats du Nord abolitionnistes, mais ses causes sont plus complexes. Elle a opposé des Etats nordistes fortement industrialisés et acquis à l'idée de la liberté individuelle à des Etats sudistes plus conservateurs, jaloux de leur indépendance, dont la richesse reposait massivement sur la culture du coton. L'industrie du Nord dépendait de l'agriculture du Sud. La question d'une union ou d'une confédération d'Etats nouveaux qui sortiraient de la conquête de l'Ouest était également présente à l'esprit. 
Les jours qui suivirent la bataille connurent souffrance et désolation, autant pour les victimes militaires que pour les civils qui avaient pratiquement tout perdu. Quelques mois après la bataille, le 19 novembre 1863, le président Abraham Lincoln vint prendre part aux cérémonies qui accompagnaient l'édification du nouveau Soldier's National Cemetery où 3.500 soldats du Nord furent enterrés (ceux du Sud furent inhumés en Caroline du Nord et en Caroline du Sud ou enterrés à Richmond, dans l'Etat de Virginie) ${ }^{1}$. Le président adressa un bref discours à la nation américaine (The Gettysburg Address) qui transformait le carnage en symbole et allait passer à la postérité : la mort de tous ces combattants ne pouvait être un vain sacrifice; elle rappelait à tous ceux qui s'étaient engagés du côté de l'Union que l'effort à faire pour vaincre définitivement était encore énorme, mais qu'il aboutirait à l'édification d'un pays de liberté pour des êtres nés dans l'égalité.

\section{Un lieu de mémoire et de réconciliation}

Durant de longues années, Gettysburg resta avant tout le lieu d'une victoire du Nord et de ses idéaux démocratiques sur le Sud conservateur. Peu à peu, il devint pour les deux camps un lieu de mémoire partagée et de réconciliation où les anciens ennemis commencèrent à se rapprocher. En 1887, à 1'occasion du 24ème anniversaire de la bataille, 1'Union des vétérans de la Brigade de Philadelphie (Nord) invita d'anciens soldats de 1 'armée des Confédérés (Sud). 1160 anciens ennemis se retrouvèrent pour célébrer ensemble l'événement. Un peu plus tard au cours de la même année, 9000 vétérans des deux camps et leurs familles devaient également se rassembler sur le champ de bataille. En 1913, à l'occasion du 50 ${ }^{\text {ème }}$ anniversaire des combats, ce sont 50.000 vétérans qui se déplacèrent vers Gettysburg. Le site était devenu le haut lieu de la réconciliation nationale et du patriotisme.

Longtemps, pourtant, de nombreux Américains allaient être tenus à l'écart des bénéfices de cette réconciliation nationale. Les anciens esclaves noirs, dont la libération avait été l'un des motifs qui avaient déclenché la guerre civile, allaient subir les affres d'une politique ségrégationniste qui ne disparaîtra qu'au milieu du vingtième siècle.

1 Notons qu'un peu plus de 3.000 autres vétérans tués au cours des guerres menées par les Etats-Unis entre 1898 - contre l'Espagne - et 1972 - Vietnam - y sont également enterrés. 
Aujourd'hui, le site de Gettysburg est vu autant comme le lieu d'un événement historique majeur pour l'Histoire des USA que comme une terre commune à l'ensemble des américains, leur « Nation's Common Ground ", sur lequel ils peuvent apprendre les enjeux idéologiques et économiques qui amenèrent à la guerre, comprendre les stratégies militaires qui permirent la victoire de l'Union et de la démocratie telle qu'elle était prônée par le Nord, découvrir en quoi ils forment une nation.

L'actuel « Gettysburg National Military Park » reçoit chaque année plus d'1,5 millions de visiteurs. Le Service des Parcs Nationaux et la Fondation Gettysburg gèrent un Centre du Visiteur qui donne notamment accès à un Musée de la Guerre Civile Américaine et à la fresque du Cyclorama de la Bataille de Gettysburg, au Cimetière National des Soldats et au champs de bataille qui s'étend sur un espace de plus de 6000 acres rendu autant que possible aux aspects qu'il offrait en 1863. 1328 monuments et plaques commémoratives - soit la plus grande collection de sculptures à ciel ouvert dans le monde - jalonnent cet ensemble mémoriel et rappellent les évènements qui s'y déroulèrent.

Le musée proprement dit a été ouvert en avril 2008. Il abrite douze galeries regroupées en trois grands ensembles proposés à la visite et à la compréhension des évènements (les causes de la guerre civile américaine et le contexte de la bataille de Gettysburg, la bataille proprement dite, les conséquences de la victoire du Nord et les résultats de la guerre). Le parcours démarre avec un film qui plonge les visiteurs dans les enjeux, les émotions et les sons de la bataille et de ses lendemains. Il se poursuit ensuite, richement agrémenté de bornes interactives, de vitrines particulièrement bien documentées, de reconstitutions scéniques, de plans et de photos qui plongent les visiteurs dans une découverte érudite de l'époque, des enjeux militaires, des souffrances humaines et des acteurs célèbres ou anonymes de cette fresque historique.

Accolé au musée, le Cyclorama de «La Bataille de Gettysburg » présente l'un des moments forts de la bataille : la charge des Confédérés de la brigade Pickett contre les retranchements des soldats de l'Union. Cette fresque exceptionnelle fut peinte en 1883-1884 par le maitre français Paul Philippoteaux secondé par un groupe de vingt artistes. Ses 377 pieds de circonférence et 27 pieds de hauteur en font avec le Panorama de Waterloo, un rare témoin d'une forme artistique de reconstitution 
qui connut une grande popularité à la charnière des XIXème et XXème siècles aux Etats-Unis comme en Europe ${ }^{1}$. Sa restauration et sa mise en valeur, après un siècle de négligence et de détérioration, en a fait un des espaces les plus prenants de la visite.

\section{Une propriété commune comme communion}

Le site de Gettysburg nous apprend qu'en matière de mémoire culturelle, l'oubli ou la mise en avant de certains évènements du passé ne suffisent pas à bâtir un pôle mémoriel intelligent et fédérateur. Il faut aussi un dessein. Les aménagements de Gettysburg servent l'idée de nation autant qu'ils en expliquent les fondements. L'accent est mis ici autant sur la grande histoire, celle des causes, de la chronologie et des grands personnages que sur les milliers d'anonymes, nés pour un grand nombre d'entre eux hors du territoire américain, qui allaient y souffrir et y perdre la vie. L'idée que le sol foulé par la masse des visiteurs soit présenté comme une commune propriété et non comme la terre d'un seul vainqueur est d'une force émotionnelle extraordinaire. Les visiteurs du lieu de mémoire sont ici chez eux. Il n'y a pas d'appropriation au profit d'un camp comme on peut le voir en d'autres endroits du monde.

Ici, la commémoration est communion, offre de connaissance autant que recueillement. Le passé est offert à la compréhension et, pour reprendre les mots de Jan Baetens, mis « au service d'une vie plus pleine, au présent comme à l'avenir ». En cela, les aménagements de Gettysburg sont le signe d'une « très haute culture » distillée sans trop d'univocité au profit du plus grand nombre.

1 Les analogies entre Gettysburg et Waterloo sont nombreuses, tant sur les évènements militaires, les manœuvres des troupes en présence, le nombre de victimes, les lendemains dramatiques que sur les enjeux nationaux. Elles le sont nettement moins, en revanche, sur les aménagements réalisés sur les deux sites. On ne peut que déplorer la pauvreté du site belge en la matière. 


\section{Bibliographie}

CANDAU, Joël, 1998, Anthropologie de la mémoire, Paris, PUF, coll. « Que saisje ? », $128 \mathrm{pp}$.

CATTON, Bruce, 2004, The Civil War, (première édition 1960, American Heritage), Boston, New York, Mariner Books, 382 pp.

GROSS, James A., COLLINS Andre B., 1991, The Souvenir Guide to the Gettysburg National Military Park, Gettysburg, Fem., 72 pp.

MOTTS, Wayne, 2008, Gettysburg Field Guide, TravelBrains, 56 pp.

MURRAY, Aaron R., (ed), 2004, Civil War. Battles and Leaders, London, New York, DK Publishing, $96 \mathrm{pp}$.

REYNIERS, Alain, 1999, "Waterloo, où la déroute de l'Aigle est gloire posthume $\mathrm{du}$ Lion " in Recherches en Communication 12 (Anthropologie des lieux de communication) : 81-96.

\section{Sites Web}

www.gettysburgfoundation.org et www.nps.gov/gett 